\title{
Carotid Endarterectomy: Techniques to Minimize the Intraoperative Risk of Embolization
}

\author{
PETER GLOVICZKI, M.D., ${ }^{1}$ WOOSUP M. PARK, M.D., ${ }^{1}$ \\ JEAN M. PANNETON, M.D., ${ }^{1}$ and KENNETH J. CHERRY, JR. M.D. ${ }^{1}$
}

ABSTRACT Embolization during carotid reconstruction is the most frequent cause of neurologic complications. Atraumatic surgical technique, intraoperative monitoring of the cerebral circulation and completion imaging all contribute to reduce the risks of embolization. Data from the literature suggest that the incidence of perioperative neurologic events correlate with the number of microemboli demonstrated by transcranial Doppler. Cerebral protection for percutaneous angioplasty with stenting of the carotid artery needs to be introduced, since without protection devices endovascular techniques are associated with $>8$ times the rate of microemboli seen during carotid endarterectomy.

Keywords Carotid endarterectomy, stroke, cerebral microemboli, carotid stenting, angioplasty

The benefit of surgical thrombendarterectomy (TEA) of the internal carotid artery depends greatly on a low rate of perioperative complications. Microemboli originating from the carotid bifurcation are the most important causes of perioperative stroke (Figs. 1 and 2). Prevention of intraoperative thrombosis and thromboembolic complications is imperative and effective techniques to minimize the risk of embolization must be mastered by all vascular surgeons. Selection of appropriate preoperative imaging study may

Perspectives in Vascular Surgery and Endovascular Therapy, Volume 14, Number 1, 2001. Address for correspondence and reprint requests: Peter Gloviczki, M.D., Division of Vascular Surgery, Mayo Clinic, 200 First St. SW, Rochester, MN 55905. ' Division of Vascular Surgery, Mayo Clinic, Rochester, MN. Copyright (c) 2001 by Thieme Medical Publishers, Inc., 333 Seventh Avenue, New York, NY 10001, USA. Tel: +1(212) 584-4662. 15310035,p;2001,14,01,107,114,ftx,en;pvs00110x. 


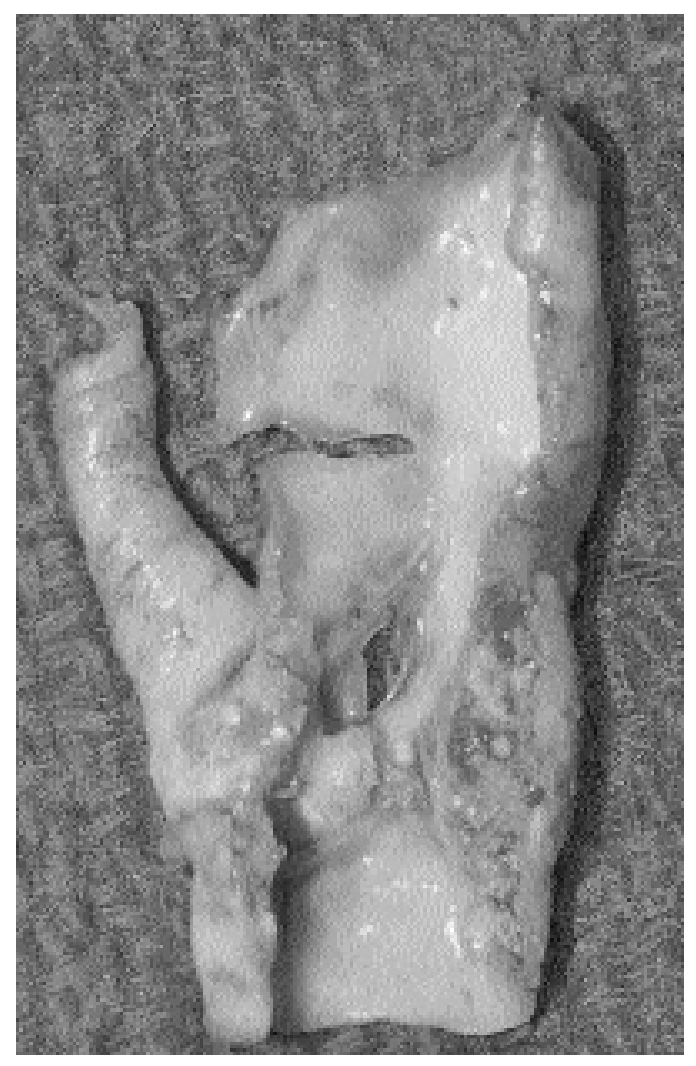

Fig. 1 Endarterectomy specimen removed during operation for high grade carotid stenosis. There is a friable atherosclerotic plaque at the origin of the internal carotid artery.

decrease the risk of preoperative embolization. During the operation, traumatic surgical technique and the use of the least traumatic instruments will decrease the risk of thromboembolism, but intraoperative monitoring of the cerebral circulation and imaging studies performed at the completion of the operation are also helpful to prevent or decrease the risk of thromboembolic complications.

\section{PREOPERATIVE IMAGING STUDIES}

Contrast arteriography with direct injection of the agent into the carotid artery carries a 1 to $6 \%$ risk of stroke, most frequently due to embolization from a friable plaque in the carotid artery. ${ }^{1}$ Duplex scanning, magnetic resonance arteriography (MRA) or 3-D computed tomography (CT) has no risk of embolization or stroke. At our institution Duplex scanning alone or with 


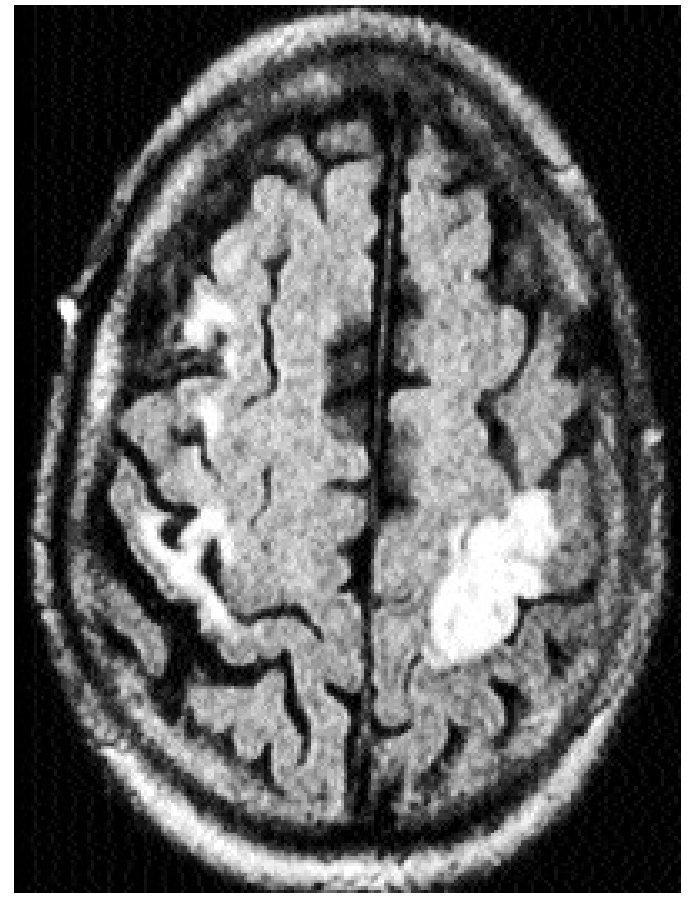

Fig. 2 Cerebral infarct in the perioperative period, not detected by electroencephalography.

MRA is used in the majority of patients who undergo carotid endarterectomy. Contrast arteriography is reserved for those patients who have contraindications for MRA, such as severe claustrophobia, have a pacemaker implanted, or for whom the MRA has been inconclusive.

\section{INTRAOPERATIVE TECHNIQUES}

\section{Positioning and Exposure of the Vessels}

Careful patient positioning, gentle rotation of the head, and avoiding firm scrubbing of the neck are all important to decrease direct trauma to the carotid bulb. Surgical dissection of the carotid arteries should be done with minimal trauma to the surrounding tissues, and direct palpation of the carotid bulb with the fingers should be avoided. We inject intravenously $5000 \mathrm{U}$ of heparin before cross-clamping, and the effect of heparin is monitored by measuring the activated clotting time (ACT) before cross-clamping. Many surgeons clamp the common carotid artery first and perform the dissection of the entire internal carotid artery only later, to decrease the risk of embolic complications. 


\section{Atraumatic Vascular Clamps}

The use of fine vascular clamps in carotid surgery is imperative (Fig. 3). For the common carotid artery many surgeons use the Fogarty clamp, which has a disposable protective surface to decrease trauma to the carotid artery. The disposable tissue contact elements consist of a fluid-filled rubber pad on one jaw and a toothed bar on the opposing bar. ${ }^{2}$ For the distal vessels, where less pressure has to be exerted, fine spring-loaded opposing DeBakey bulldogs can be used, although small clamps with a disposable protective surface are also available. Moore et al. reviewed the types and extent of intimal and medial injury that can be caused by vascular clamps. ${ }^{3}$ They proposed a 5-level classification scheme for clamp injuries, from no injury through mural integrity disruption. Endothelial injury leads to platelet aggregation, adhesion, and thrombus formation, which serves as nidus for embolic complications. Early occlusive thrombosis or late intimal hyperplasia are additional potential complications of clamp injuries. Moore et al. ${ }^{4}$ measured the grade of intimal injury caused by different vascular clamps. The authors found a direct correlation between the magnitude of transmural force and resultant intimal injury. The Fogarty soft jaw clamp caused the least amount of intimal

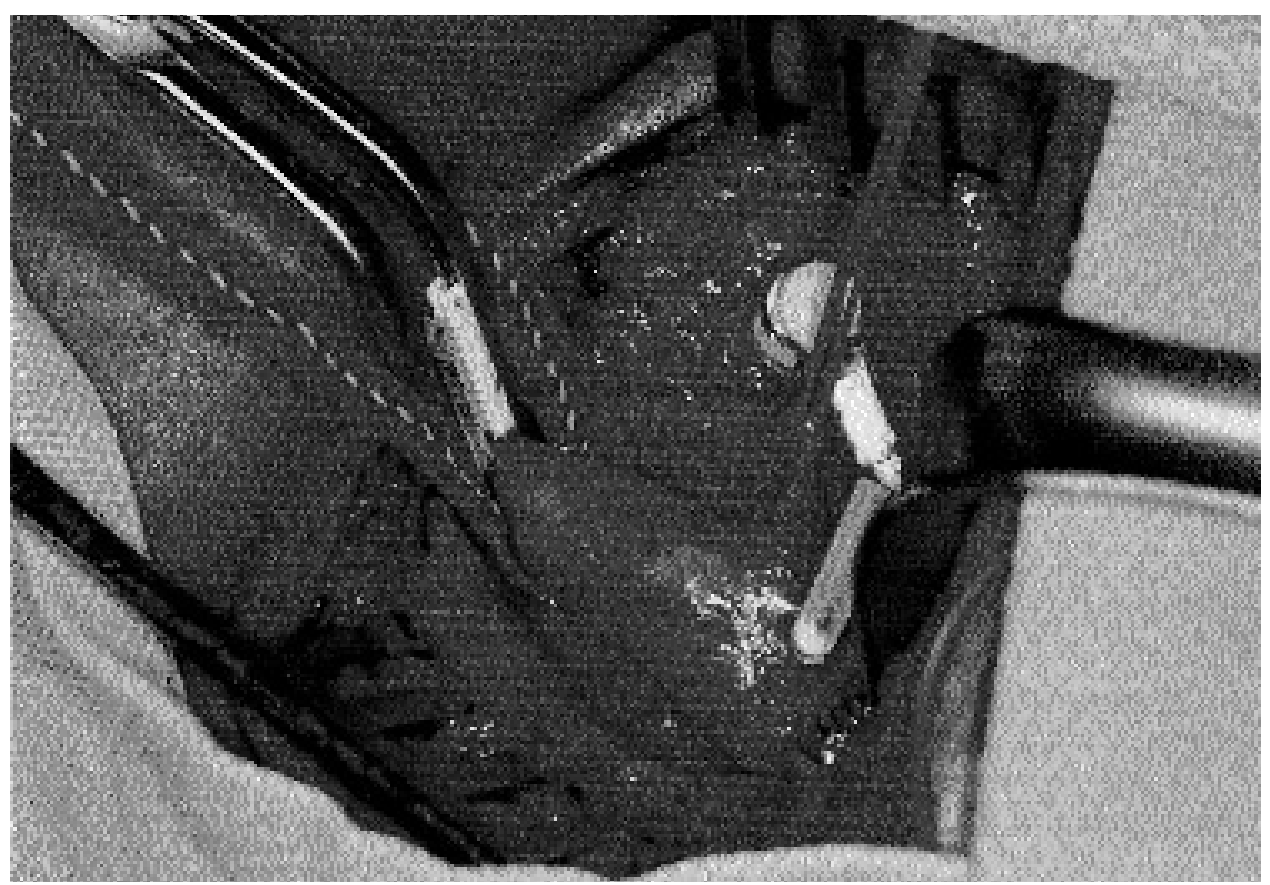

Fig. 3 Intimal trauma can be minimized by atraumatic vascular clamps. Note a soft, compliant elastomeric pad molded on a semi-rigid plastic base. (Novare Surgical Systems, Cupertino, CA.) 
injury, likely due to a very compliant contact surface of the soft-jaw inserts, which disperses the force more evenly between the jaws.

\section{Carotid Shunts}

The use of internal shunts prevents stroke in those patients who have poor intracerebral collaterals or who have significant occlusive disease in the contralateral carotid and the vertebral arteries. Shunts, however, are also potential causes of air and atheroemboli. In addition, they can cause intimal injury and may make the distal endpoint of the endarterectomy difficult to visualize. Selective shunting, therefore, is strongly recommended to decrease the need for shunting. Intraoperative EEG monitoring, operation on an awake patient using cervical block, measurement of carotid artery stump pressure, Xenon cerebral blood-flow measurements, the use of somatosensory evoked potential measurements, and transcranial Doppler are the techniques most frequently used to determine the adequacy of cerebral circulation during carotid clamping. Although most of these techniques are effective, emboli can occur in spite of sophisticated intraoperative monitoring or preventive measures

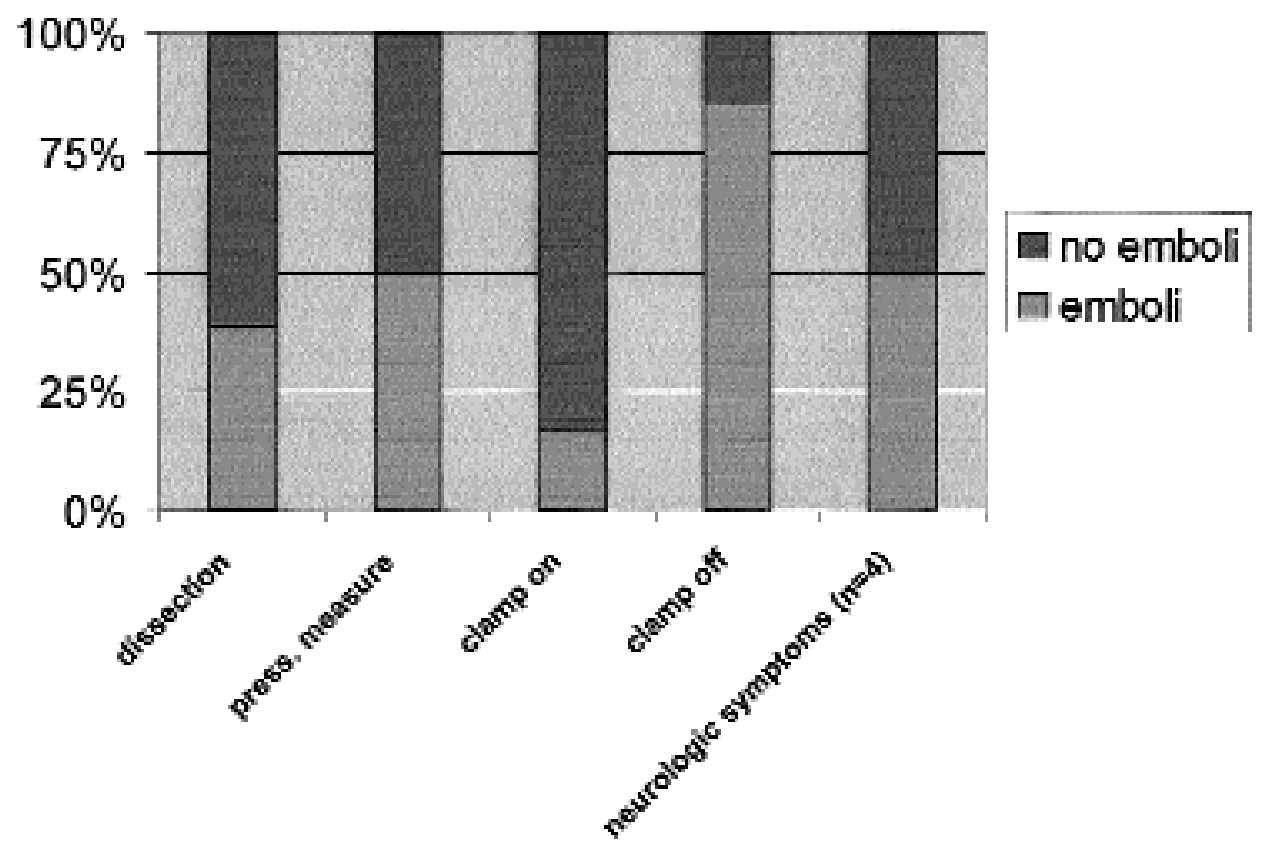

Fig. 4 The incidence of cerebral microemboli measured with transcranial Doppler during 61 carotid endarterectomies. (Data compiled from Gao MY, Sillesen HH, Lorentzen JE, Schroeder TV. Eversion carotid endarterectomy generates fewer microemboli than standard carotid endarterectomy. Eur J Vasc Endovasc Surg 2000;20:153-157.) 
(Fig. 2). To decrease embolization to a minimum, we use a small Sundt polyethylene shunt, which has a round, smooth tip to minimize endothelial injury. The proximal end in the common carotid artery is secured first with a modified Rumel tourniquet and the Fogarty clamp is released for a second to flush all air and debris from the artery and the shunt. The distal end is placed second, during back bleeding from the internal carotid artery to avoid air embolism. A fine rubber loop is used to secure the shunt distally in the internal carotid artery.

The risk of shunting was assessed in a study of 500 patients who underwent carotid endarterectomy. Using transcranial Doppler, Spencer found that 13 of the 24 intraoperative strokes were caused by embolization. ${ }^{5}$ Although the number of shunted patients with embolization was similar to the nonshunt patients, all those who had stroke due to hyperperfusion had shunts. Spencer found that shunts are associated with complications, and recommended using transcranial Doppler to monitor and decrease the risk of intraoperative embolism.

\section{Imaging Studies at Completion of TEA}

Contrast arteriography, angioscopy, and intraoperative Duplex scanning have been used to exclude partial or complete thromboses, intimal flaps, dissection, or residual stenosis in the carotid vessels. Panneton et al., from our institution, studied the benefits of intraoperative Duplex scanning. 6 Fortyseven $(30 \%)$ of 155 examinations performed on 45 patients had minor abnor-

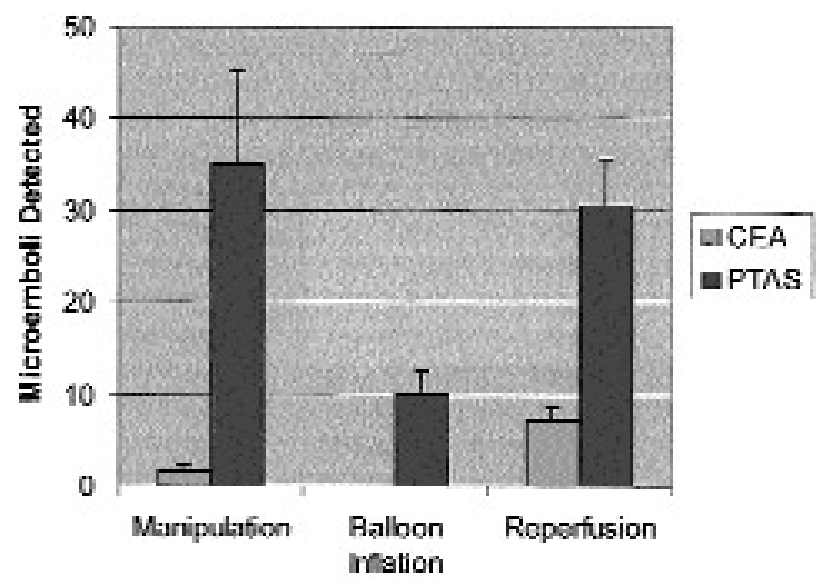

Fig. 5 Cerebral microembolization during surgical endarterectomy (CEA) and carotid angioplasty and stenting (PTAS), measured with transcranial Doppler. (Data from Jordan WD, Voellinger DC, Doblar DD, Plyushcheva NP, Fisher WS, McDowell HA. Microemboli detected by transcranial Doppler monitoring in patients during carotid angioplasty versus carotid endarterectomy. Cardiovasc Surg 1999;7:33-38.) 
malities consisting of insignificant residual plaque; residual external carotid artery stenoses; small intimal flaps; elevated velocities with no associated anatomic lesion; and an arterial kink. Fourteen patients (9\%) had significant findings requiring immediate surgical revision. These consisted of large intimal flaps or dissection in six patients; marked residual plaque and significant stenosis in five patients; thrombus in two patients; and a kink in one patient. Three additional patients $(2 \%)$ had significant findings but were not revised. No difference was identified in outcome among patients with normal exams, those with minor technical defects, and those with significant abnormalities undergoing surgical revision. However, two out of three patients who had significant abnormalities within the common carotid artery that were not revised suffered perioperative ipsilateral strokes. We concluded that repair of significant technical defects will likely decrease perioperative thromboembolic complications of carotid TEA.

\section{EMBOLIZATION DURING SURGICAL TEA VERSUS CAROTID STENTING}

Available data in the literature suggest that carotid angioplasty and stenting has a significantly higher rate of cerebral embolization than carotid TEA. Jordan et al. reviewed results of 112 procedures for the treatment of 115 carotid bifurcation stenoses: 40 by percutaneous angioplasty with stenting and 75 by carotid endarterectomy. ${ }^{7}$ Transcranial duplex scanning was used during each procedures to monitor microembolization. During angioplasty and stenting, there was a mean of 74.0 emboli per patient (range 0 to $398, P=0.0001$ ), and 4 neurologic events occurred $(P=.08)$. During endarterectomy, a mean of 8.8. emboli occurred per patient (range 0 to $102, P=0.0001$ ), and one had a neurologic event $(P=0.08)$. Two patients developed stroke $(5.6 \%)$, and 2 had transient ischemic deficit (5.6\%) after stenting, and the number of microemboli in these cases was $133,17,29$, and 47 , respectively. The only patient who developed stroke after carotid endarterectomy (1.4\%) had 48 microemboli noted during the procedure. Only 3 patients $(7.5 \%)$ had no emboli after stenting, whereas 29 carotid endarterectomy patients had no emboli $(38.7 \%)$. The authors concluded that percutaneous angioplasty with stenting of the carotid artery is associated with more than eight times the rate of microemboli seen during carotid endarterectomy when evaluated with transcranial Doppler monitoring. Effective devices for cerebral protection should urgently be introduced to decrease the high rate of microemboli noted during carotid angioplasty and stenting.

\section{REFERENCES}

1. Ricci MA, Knight SJ. The role of non-invasive studies in the diagnosis and management of cerebrovascular disease. In: Rutherford RB, ed. Vascular Surgery, 5th ed. Philadelphia: WB Saunders; 2000:1775-1789 
2. Fogarty TJ, Raible DA, Krippaehne WW. A new vascular clamp. Am J Surg 1966; 112:966-968

3. Moore WM Jr, Bunt TJ, Hermann GD, Fogarty TJ. Assessment of transmural force during application of vascular occlusive devices. J Vasc Surg 1988;8(4):422-427

4. Bunt TJ, Manship L, Moore W. Iatrogenic vascular injury during peripheral revascularization. J Vasc Surg 1985;2(3):491-498

5. Spencer MP. Transcranial doppler monitoring and causes of stroke from carotid endarterectomy. Stroke 1997;28:685-691

6. Panneton JM, Berger MW, Lewis BD, et al. Intraoperative Duplex ultrasound during carotid endarterectomy. Vasc Surg, 2000, in press

7. Jordan WD Jr, Voellinger DC, Doblar DD, et al. Microemboli detected by transcranial Doppler monitoring in patients during carotid angioplasty versus carotid endarterectomy. Cardiovasc Surg 1999;7(1):33-38 